\title{
EFFECTS OF SOMATOSTATIN ON MAMMALIAN CORTICAL NEURONS IN CULTURE: PHYSIOLOGICAL ACTIONS AND UNUSUAL DOSE RESPONSE CHARACTERISTICS ${ }^{1}$
}

\author{
JOHN R. DELFS ${ }^{2}$ AND MARC A. DICHTER \\ Departments of Neurology, Harvard Medical School and Beth Israel Hospital, and Division of Neuroscience, The Children's \\ Hospital Medical Center, Boston, Massachusetts 02115
}

Received November 5, 1982; Revised January 25, 1983; Accepted January 28, 1983

\begin{abstract}
Somatostatin was applied to rat cortical neurons grown in dispersed cell culture while electrophysiological effects were monitored with intracellular recordings. The effects of somatostatin were predominantly excitatory, but they varied and were dose dependent. Somatostatin at concentrations between 100 pM and $1 \mu \mathrm{M}$ depolarized 30 of 87 neurons, often with a concomitant increase in spontaneous action potentials and a small increase in membrane input resistance. Somatostatin also increased the frequency of synaptic activity coming into the recorded neuron in 45 of 78 recordings, an effect which was seen even in the absence of direct effects. In six of nine neurons, somatostatin decreased the amplitude of large ongoing inhibitory synaptic potentials, concomitant with an increase in membrane input resistance. Somatostatin occasionally caused inhibition of neuronal activity, an effect predominantly associated with the application of higher concentrations. The unpredictability of the response of an individual neuron to somatostatin is partially explained by three phenomena: a marked tachyphylaxis, an inverted U-shaped dose response curve for membrane depolarization, and qualitatively different responses depending on the concentration of somatostatin. These findings show that somatostatin is predominantly an excitatory agent when applied to cortical neurons, but it may cause several different changes in membrane properties. The dose response data suggest a considerable complexity of the actions of somatostatin at a cellular level.
\end{abstract}

Somatostatin is a 14-amino acid cyclic polypeptide having a number of characteristics compatible with a role in neuronal transmission in the cerebral cortex. It was originally detected in hypothalamic extracts as a factor which inhibited the release of growth hormone from pituitary cells in culture (Krulich et al., 1968) and was later isolated, characterized, and synthesized, with demonstration of bioactivity equivalent to the hypothalamic factor (Brazeau et al., 1973). However, although the concentration of somatostatin is highest in the hy-

${ }^{1}$ This work was supported by The Esther A. and Joseph Klingenstein Fund, Grants NS15362, NS00130, NS06320, NS00608, and The Children's Hospital Medical Center Mental Retardation Core Grant HD06276. We thank Bernard Biales and Sara Vasquez for expert technical assistance and Diane Kilday for help with preparation of the manuscript. We thank W. Frost White, Deborah Barnes, and Michael Selzer for thoughtful comments on this manuscript. We are grateful to Seymour Reichlin for providing the anti-somatostatin immune serum and to him and Judy Gruber for somatostatin radioimmunoassays on innumerable samples.

${ }^{2}$ To whom correspondence should be addressed at Division of Neuroscience, Enders 1, The Children's Hospital Medical Center, 300 Longwood Avenue, Boston, MA 02115. pothalamus, the cerebral cortex contains a larger total amount of somatostatin than any other region of the nervous system (Patel and Reichlin, 1978), and this cerebral cortical somatostatin appears to arise from intrinsic neurons (Martin et al., 1978; Delfs et al., 1980). A large proportion of the immunoreactive somatostatin is concentrated in purified synaptosomal fractions (Berelowitz et al., 1978) and its release is calcium dependent (Iversen et al., 1978; Lee et al., 1979). A specific high affinity receptor for somatostatin recently demonstrated in rat brain membranes (Srikant and Patel, 1981a) has characteristics which are different from the somatostatin receptor in the pituitary (Srikant and Patel, 1981b).

There is evidence to suggest that somatostatin may play a role in mammalian behavior and in several neurological disease states. Somatostatin has dramatic behavioral effects when applied in vivo (Plotnikoff et al., 1974; Rezek et al., 1977), and its relation to seizure disorders has been suggested (Havlicek et al., 1975). Also, somatostatin levels have been found to be significantly depressed in the brains of patients with senile dementia of the Alzheimer type (Davies et al., 1980).

Numerous groups have reported effects of somatosta- 
tin on the electrophysiological activity of central neurons (Renaud et al., 1975; Miletic et al., 1977; Nicoll, 1978; Padjen, 1977; Ioffe et al., 1978; Olpe et al., 1980; Macdonald and Nowak, 1981), but specific results have often been conflicting.

In the present studies, intracellular recordings were made from individual neurons in cultures of fetal rat cortex while known concentrations of somatostatin were applied by microperfusion. We have previously shown that neurons in these cultures produce and secrete somatostatin (Delfs et al., 1980). We describe below that somatostatin affects the physiological functioning of a cortical neuron by apparently direct effects on the membrane of the recorded cell itself or by indirect effects on neurons presynaptic to the recorded neuron. The response is often complex and is most often excitatory. However, we have also observed that in some recordings somatostatin application causes a diminished neuronal excitability or a decrease in the frequency of synaptic activity. These observations have provided an opportunity to examine both excitatory and inhibitory responses to somatostatin in the same system.

The present report demonstrates that the types and magnitudes of responses seen in individual neurons are related both to the concentration of the applied somatostatin and to the prior history of somatostatin exposure. These results suggest possible explanations for the seemingly disparate results of previous studies on the physiological actions of somatostatin on central neurons. Preliminary reports of these data have been presented (Delfs et al., 1979, Delfs and Dichter, 1981).

\section{Materials and Methods}

Dissociated cell cultures of rat cerebral neurons were prepared as reported by Dichter (1978) and subsequently modified (Snodgrass et al., 1980). Pregnant CD rats, at 15 to 16 days postconception, were stunned and embryos removed by sterile dissection. Cortices were dissected, incubated successively in trypsin and calcium-, magnesium-free Earle's balanced salt solution, and then triturated, filtered, and plated at about 250,000 cells $/ 35-\mathrm{mm}$ diameter Petri dish in modified minimal essential medium containing $D$-glucose $(200 \mathrm{mg} / 100 \mathrm{ml})$, penicillin (20 units $/ \mathrm{ml})$, streptomycin $(20 \mathrm{mg} / \mathrm{ml})$, rat serum $(5 \%)$, and methylcellulose $(200 \mathrm{mg} / 100 \mathrm{ml})$. Cultures were grown in plastic tissue culture dishes (Costar, Falcon) coated in advance with collagen and polylysine. Medium was changed at $24 \mathrm{hr}$ and three times per week thereafter. Non-neuronal cell growth was inhibited with cytosine arabinoside for $24 \mathrm{hr}$ when background cells reached confluency. Studies were performed between 3 and 12 weeks after plating.

For intracellular recordings, the cortical neurons were bathed in a physiological salt solution, visualized under an inverted phase contrast microscope, and impaled with $3.5 \mathrm{M}$ potassium acetate-filled microelectrodes (40 to 80 megohms). Recordings were made with a standard amplification arrangement (WPI 701). A modified Wheatstone bridge was used to pass current through the electrode while recording the potential across the cell. Membrane potential and the amount of current injected were recorded with a penwriter and displayed on an oscillo- scope for photographic recording. Criteria for accepting a neuron for analysis included a stable resting membrane potential of at least $-40 \mathrm{mV}$ and an evoked action potential with a height of at least $50 \mathrm{mV}$. Neurons were characterized by the resting membrane potential $\left(V_{m}\right)$ and the size, duration, frequency, and pattern of synaptic potentials and spontaneous action potentials (APs). Measurements were made of the change in $V_{m}$ in response to regularly spaced injections of 50 -msec square wave pulses of hyperpolarizing current and the membrane input resistance $\left(R_{\text {in }}\right)$ was determined using Ohm's law. Action potentials were evoked by pulses of depolarizing current, and AP height, duration, and number of APs per depolarizing pulse were measured.

Synthetic cyclic somatostatin-14 from several sources (Bachem, Beckman, and Peninsula Laboratories) was dissolved in a phosphate-buffered physiological salt solution with $0.1 \%$ bovine serum albumin (BSA) (Sigma, radioimmunoassay grade) to optimize peptide stability. Aliquots of known concentrations of somatostatin, all with identical concentrations of BSA, were then kept frozen until use. At the time of each experiment, the somatostatin solution was loaded into micropipettes (tip diameter $5 \mu \mathrm{m}$ to $15 \mu \mathrm{m}$ ) and perfused locally over the neuron under study. Concentrations of somatostatin were verified prior to use and at the completion of experiments by a well characterized radioimmunoassay (Patel and Reichlin, 1978). For the initial parts of the study, somatostatin was applied at concentrations of $10^{-10}$ to $10^{-6} \mathrm{M}$. For the dose response studies, the concentration range was extended to include concentrations of somatostatin from $10^{-11}$ to $10^{-3} \mathrm{M}$. All test solutions contained the $0.1 \%$ BSA, and control solutions were identical to test solutions except for the absence of somatostatin.

\section{Results}

\section{Depolarization, excitation, and increased membrane input resistance}

Depolarization. Somatostatin caused a membrane depolarization (mean $5.9 \pm 3.4 \mathrm{mV}$ ) in 30 of 87 neurons $(34 \%)$ when applied at concentrations from $10^{-10}$ to $10^{-6}$ M (Fig. 1). The onset of the depolarization was rapid (within $1 \mathrm{sec}$ of the onset of the perfusion), but could last for several seconds to as long as $10 \mathrm{~min}$ after termination of the perfusion. The size of the depolarization was not related to the base line input resistance, the amount or type of ongoing synaptic activity, the pattern of neuronal firing, or the age of the culture. The depolarizing response to somatostatin application was not prevented by tetrodotoxin (TTX) (Fig. 1D). It could not be determined from the present data whether TTX attenuated this effect of somatostatin.

Excitation. In 12 neurons which had spontaneous APs and were also depolarized by somatostatin there was an associated increase in the frequency of APs in nine (75\%). This was attributable to an increase in EPSPs in six neurons and to the depolarization itself in the absence of EPSPs in three neurons. Somatostatin also increased neuronal excitability without a direct depolarization by increasing the number of APs evoked by a square wave pulse of depolarizing current (in 7 of 21 neurons tested) 

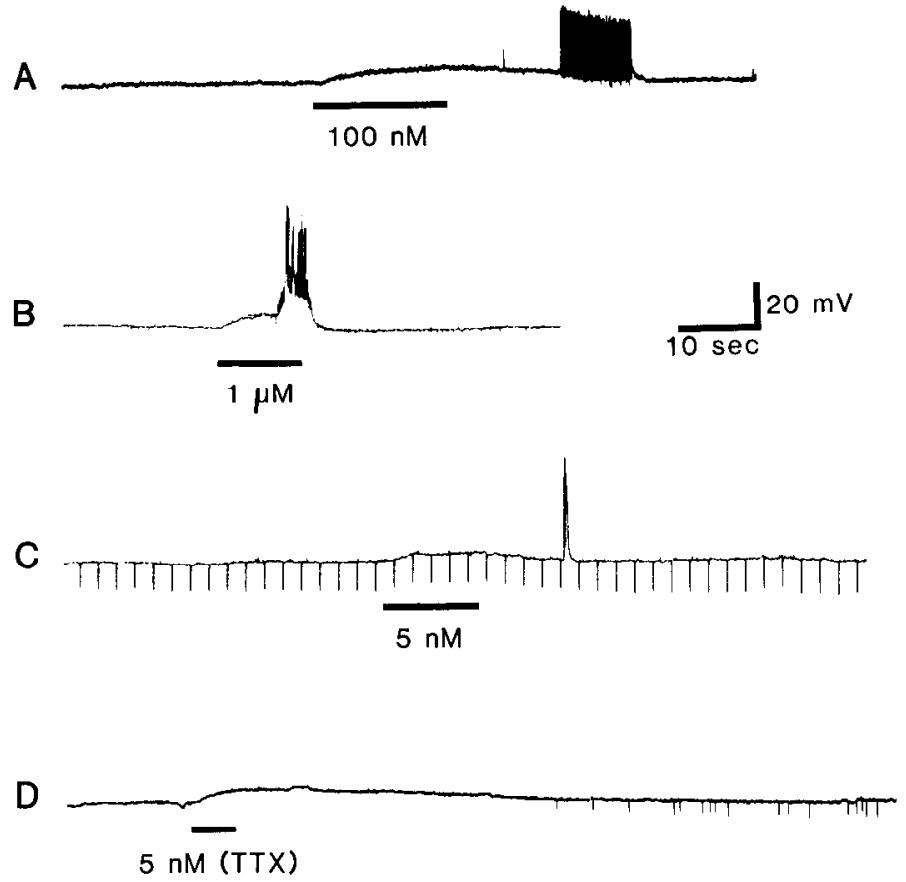

Figure 1. Depolarization of four different cortical neurons by somatostatin. $A$, Membrane depolarization in a neuron with little detectable synaptic activity, with a delayed volley of action potentials (APs) occurring during the recovery phase. $B$, An 8-mV depolarization is the initial response to somatostatin. A burst of incoming excitatory activity and associated action potentials is superimposed on the depolarization with a longer latency of onset. $C$, Depolarizetion of neuron into which pulses of hyperpolarizing current are being injected at regular intervals shows no measurable alteration of membrane input resistance $\left(R_{\text {in }}\right)$ during the depolarization, but a slight increase in $R_{\text {in }}$ can be appreciated after the depolarization has subsided. $D$, In another neuron, depolarization still occurs with the application of somatostatin in the presence of tetrodotoxin $(T T X)\left(3 \times 10^{-7}\right.$ M). (The small hyperpolarizing potentials occurring at the end of the tracing in $D$ represent spontaneous IPSPs which occurred after the TTX had diffused away into the bath.) Resting membrane potentials $\left(V_{m}\right)$ were $-68,-76,-62$, and $-64 \mathrm{mV}$, respectively. APs in this and subsequent figures are significantly attenuated by the penwriter.

or by increasing the frequency of ongoing spontaneous APs (Fig. 2).

Increased membrane input resistance $\left(R_{\mathrm{in}}\right) . R_{\text {in }}$ was monitored during microperfusion with somatostatin test solutions in 37 neurons and was observed to increase in 17 of these neurons $(46 \%)$ with the mean $R_{\text {in }}$ increasing from 43 to 51 megohms. This effect was seen both with and without the depolarizing response to somatostatin. There were concomitant increases in membrane resistance in 4 of the 11 neurons which were also depolarized by somatostatin, and in 13 of the 26 neurons which were not depolarized. In no case was there an decrease in $R_{\text {in }}$ in response to somatostatin within this concentration range.

Dose response analyses. For these dose response analyses the dosage range was extended to include concentrations from $10^{-11}$ to $10^{-3} \mathrm{M}$. Two factors, the greal variability in the magnitude of the response among individual neurons and a prominent tachyphylaxis, prevented an adequate analysis of the amplitude of depolar- ization as a function of concentration. These factors necessitated analyses which would minimize interneuronal differences and emphasize interdose differences, while dealing with a wide range of concentrations. We have, therefore, presented the dose response data for somatostatin-associated depolarizations in two ways.

Figure 3 indicates the proportion of neurons depolarized ( $1 \mathrm{mV}$ or greater) when exposed to somatostatin at various concentrations. This analysis demonstrates a bimodal distribution of responsiveness of neurons to the depolarizing effect of somatostatin. The threshold concentration was between 10 and $100 \mathrm{pM}$. Concentrations in the range of $100 \mathrm{pm}$ to $5 \mathrm{~nm}$ were associated with a $30 \%$ to $40 \%$ response rate. Further increases in somatostatin concentration (10 $\mathrm{nM}$ to $1 \mu \mathrm{M})$ resulted in a decrease in responsiveness. At still higher concentrations ( $10 \mu \mathrm{M}$ to $1 \mathrm{mM}$ ), depolarizations occurred in an increasing percentage of neurons. However, the depolarizations at these highest concentrations were in many cases different from those seen at the lower concentrations in that they were often greater than $30 \mathrm{mV}$, were associated with a marked conductance increase (as opposed to the usual slight decrease or no change in conductance), and were often irreversible. Thus, some of the effects of somatostatin at and above $10 \mu \mathrm{M}$ may represent either a different type of depolarizing response or a toxic effect.

A second method of analysis was to construct a composite diagram of the depolarizations seen in individual neurons in response to the two different concentrations of somatostatin used in each particular experiment (Fig. 4). This allows the graphic representation of differences in the amplitude of the depolarization in response to different concentrations of somatostatin without obscuring the interneuronal differences. This analysis also shows three phases in the dose response relationship. In the initial phase (10 pM to $100 \mathrm{pm}$ and $500 \mathrm{pm}$ to $5 \mathrm{nM}$ ), increasing concentrations of somatostatin produce larger depolarizations in 16 of 16 neurons (100\%). In marked contrast, however, increasing the somatostatin concentration from $5 \mathrm{~nm}$ to $500 \mathrm{~nm}$ produces an increase in the amplitude of the depolarization in only 4 of 16 neurons $(25 \%)$ and a decreased depolarization in response to the application of the larger concentration in 12 neurons $(75 \%)$. As the concentration is increased from $1 \mu \mathrm{M}$ to 1 $\mathrm{mM}$ there is an increase in the depolarization amplitude in the majority of neurons tested, but, as noted above, this last phase probably represents a qualitatively distinct depolarization or toxic effect.

Within the concentration range from $500 \mathrm{pm}$ to $500 \mathrm{nM}$, the phenomenon of a larger response to the lower of two concentrations of somatostatin is often striking (Fig. 5). These records of individual neuronal responses are representative of the data from the above analyses. The reproducibly larger responses from lower concuntrations rule out the possibility that the order in which the doses were tested is responsible for this phenomenon.

\section{Increased synaptic activity}

Response characleristics. The most frequently observed effect of somatostatin was an increase in the synaptic activity within the neuronal circuit being monitored through the recorded neuron. In 78 neurons having 
A

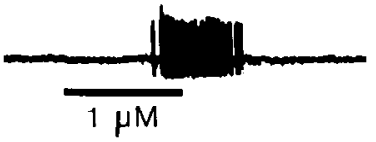

B

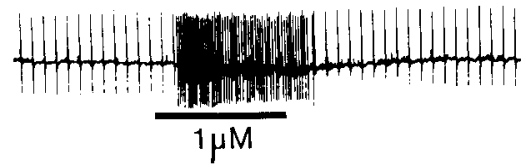

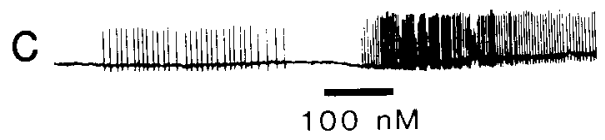

D 1

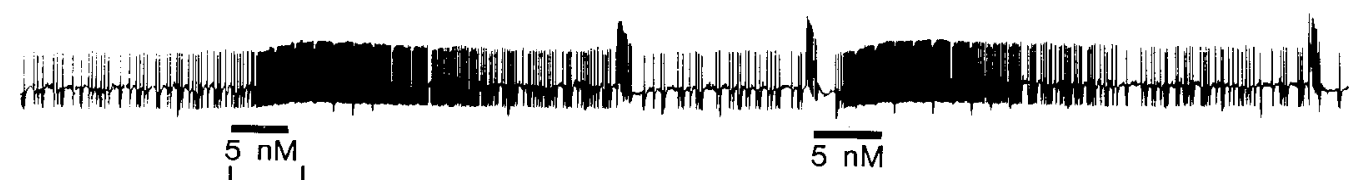

D2

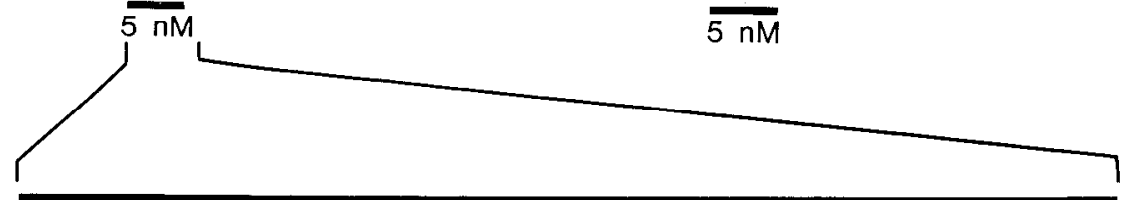

2
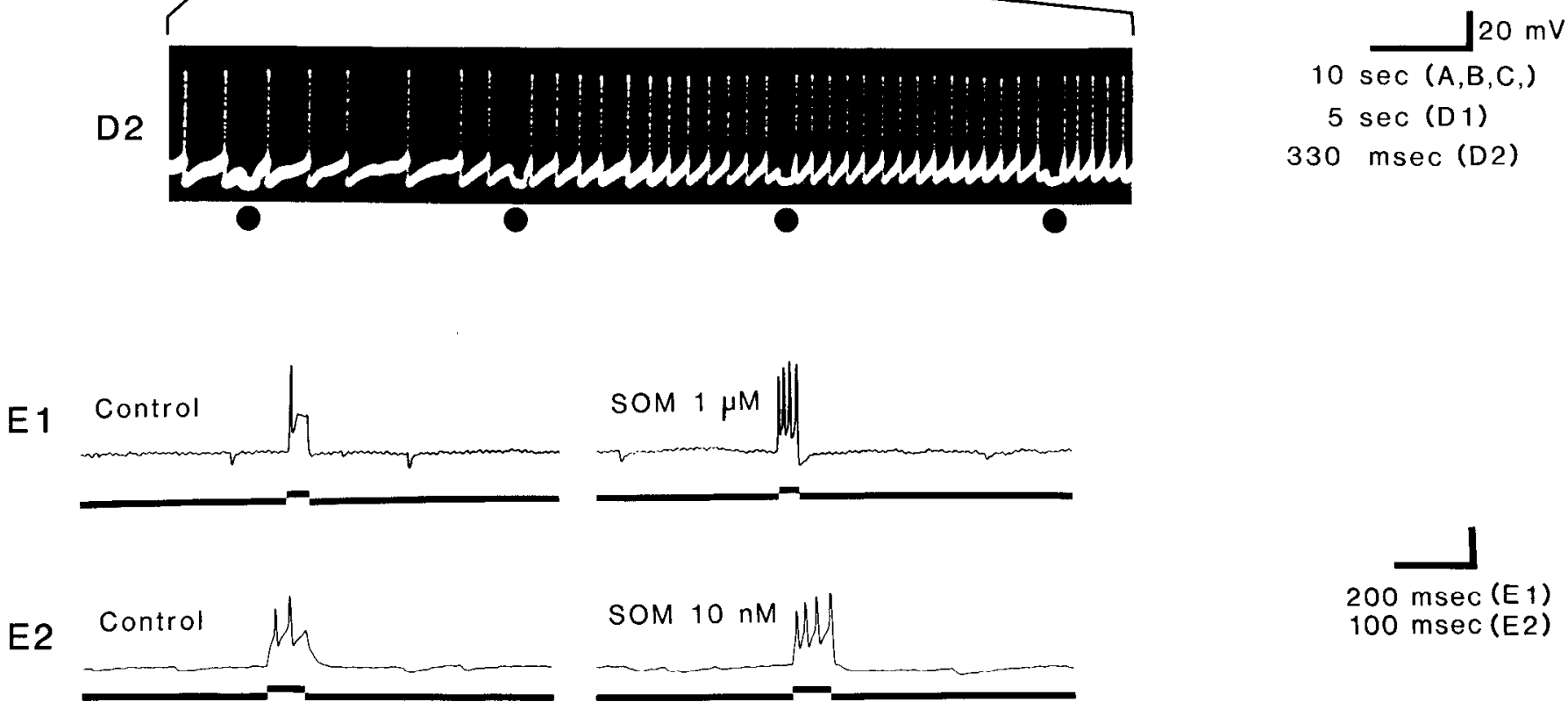

Figure 2. Excitability changes in cortical neurons with application of somatostatin. $A$, Burst of spontaneous APs with no change in membrane potential. $B$, Neuron recorded with regular APs evoked by pulses of depolarizing current (current trace not shown) responds with a burst of APs despite slight hyperpolarization (also seen with control as a small perfusion artifact). $C$, A slightly delayed and prolonged volley of APs despite slight perfusion-associated hyperpolarization (as in B). D1, dramatic increase in frequency of APs in a spontaneously firing neuron in response to somatostatin. D2, Segment of the oscilloscope tracing concomitant with the penwriter record of the same neuron as in $D 1$ shows no actual depolarization of the base line $V_{m}$ or any change in the AP threshold, but rather an increase in the slope of the depolarizing phase leading to AP initiation and a decrease in the amplitude of the afterhyperpolarizations. The black dots represent times of injection of hyperpolarizing current during the recording. E1 and E2, somatostatin application increases the number of APs evoked by pulses of depolarizing current in neurons which showed no depolarization. (AP heights are inaccurate, except in $D 2$, due to attenuation by the penwriter.) Resting $V_{m}$ for $A$ through $E 2$ : $-54,-78,-70,-56,-68$, and $-66 \mathrm{mV}$, respectively.

spontaneous postsynaptic potentials (PSPs) during a base line recording, the application of somatostatin within the dosage range of $10^{-10}$ to $10^{-6} \mathrm{M}$ resulted in an increase in PSP frequency in 45 neurons (58\%). The frequency of both excitatory postsynaptic potentials (EPSPs) and inhibitory postsynaptic potentials (IPSPs) was increased by somatostatin (Fig. 6). In any given neuron with both EPSPs and IPSPs, one or the other population of PSPs was usually increased preferentially, but the ratio of EPSPs to IPSPs was not altered in any consistent direction. In most instances there was a latency of 2 to $10 \mathrm{sec}$ from the onset of the somatostatin perfusion until the beginning of this response. Once the increase in PSP frequency occurred, it could persist for $60 \mathrm{sec}$ or more after the cessation of perfusion (Fig. $6 \mathrm{D}$ ).

Dose response analysis. For the dose response analy- sis, the dosage range was again extended to include concentrations of somatostatin from $10^{-11}$ to $10^{-3} \mathrm{M}$. The effect of somatostatin to increase the frequency of PSPs in neuronal circuits was seen throughout this entire dosage range (Fig. 7). In contrast to the dose response characteristics of the somatostatin-associated depolarization, the frequency of synaptic activity was progressively more likely to be increased with higher concentrations of somatostatin. Nevertheless, in individual recordings, there was sometimes a greater increase in the frequency of PSPs with the lower concentration of somatostatin.

The effect of somatostatin to increase the PSP frequency is probably due to an action of somatostatin on neurons presynaptic to the impaled neuron. Thus, the monophasic dose response relationship over many orders 


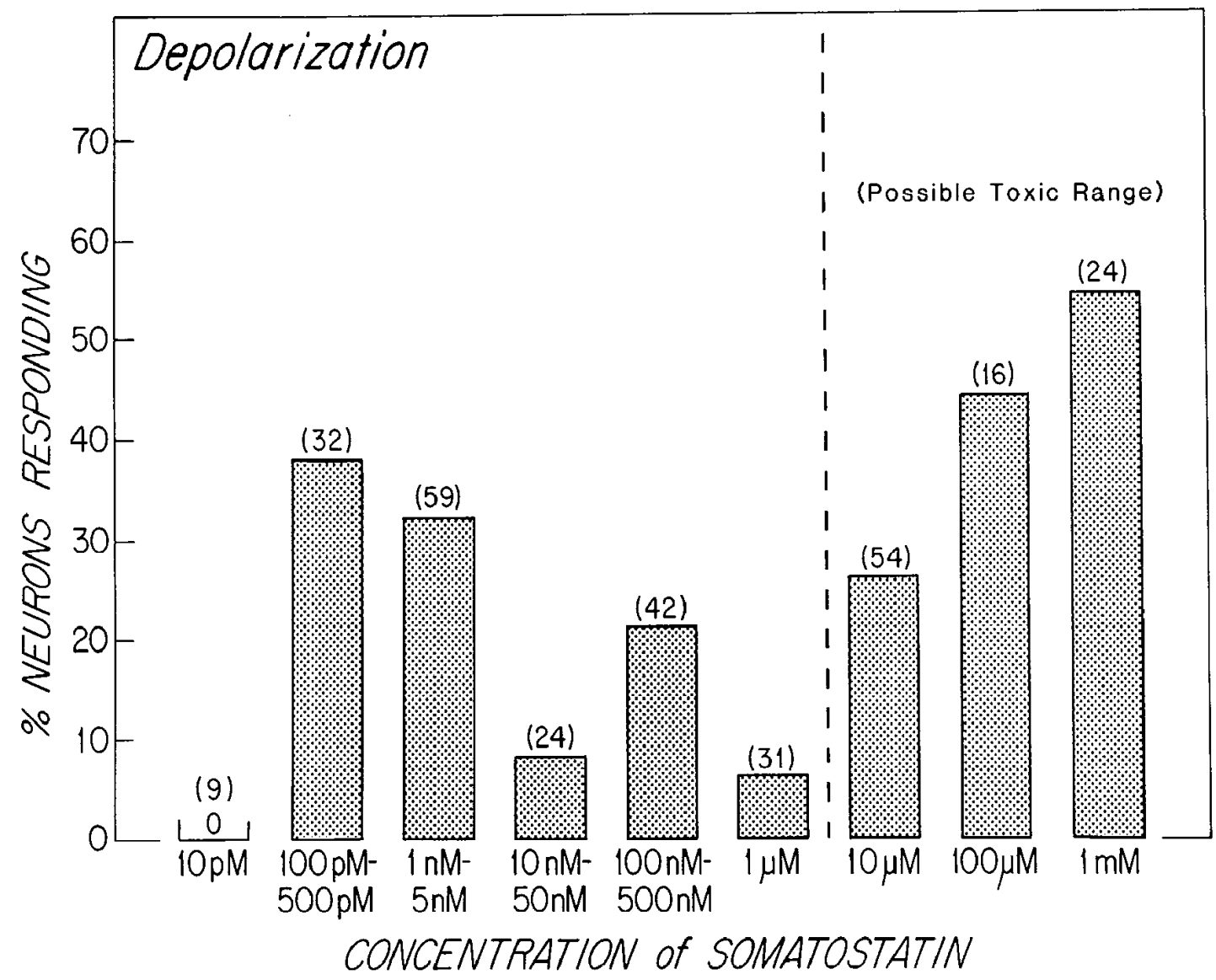

Figure 3. Percentage of tested neurons responding with a depolarization to the application of somatostatin in concentration groupings from $10 \mathrm{pM}$ to $1 \mathrm{mM}$. The number of neurons tested in each concentration range is noted in parentheses. From 10 pM to the mid- and high nanomolar range there is an inverted-U shape to this relationship with a rising phase from $10 \mathrm{pm}$ to 100 to $500 \mathrm{pM}$ and an apparent falling phase from 100 to $500 \mathrm{pM}$ to $10 \mathrm{nM}$ to $1 \mu \mathrm{M}$. The late rising phase from $10 \mu \mathrm{M}$ to $1 \mathrm{mM}$ (noted as possible toxic range) represents in part a qualitatively different response characterized by long lasting, and often irreversible, depolarizations of large magnitude often associated with a marked decrease in $R_{\text {in }}$.

of magnitude of somatostatin concentration may be due to higher applied concentrations producing threshold concentrations at distances farther from the recording site, thereby increasing the likelihood of exciting some neuron within the local circuit.

\section{Tachyphylaxis}

Whether the response to the application of somatostatin was a depolarization or an increase in the synaptic activity, successive applications of somatostatin resulted in a stepwise diminution in the magnitude of these responses until the responses were no longer recorded (Figs. 8 and 10B). The decrement in the response magnitude occurred more quickly with higher concentrations or when the interval between applications was shorter. A partial recovery of responsiveness was regularly obtained after a 2- to 10-min somatostatin-free interval, but even after these intervals the application of the same or a higher concentration of somatostatin only rarely resulted in a response magnitude as great as that seen on initial application.

\section{Alterations in the amplitude of PSPs}

Although the usual PSP response to somatostatin was an increase in PSP frequency with no measurable change in PSP amplitude, in a number of recordings definite decreases in IPSP amplitudes were noted. This effect was analyzed in nine neurons which had ongoing IPSPs with stable amplitudes of at least $4 \mathrm{mV}$ and in which the application of somatostatin caused neither a depolarization nor a significant alteration in the frequency of synaptic activity. In six of these nine neurons, the amplitude of the IPSPs was significantly diminished (Fig. 9). In four of these neurons the $R_{\text {in }}$ was monitored, and in all four cases the decrease in IPSP amplitude was associated with a concomitant increase in $R_{\text {in. }}$. Both the decreased amplitude of the IPSPs and the increase in $R_{\text {in }}$ were reversible over seconds to minutes upon termination of the somatostatin perfusion.

\section{Inhibitory responses}

Inhibition as the predominant response. Although excitation was the response seen most commonly, somatostatin application within this dosage range sometimes resulted solely in an inhibitory response. In 9 of 87 recordings the inhibitory response was the predominant response; of these, there was a decreased frequency of spontaneous APs in two recordings, a decreased number of APs per depolarizing pulse of current in four recordings, and a decrease in the frequency of PSPs in three recordings. Therefore, inhibition was the predominant response in a small percentage of the total number of 


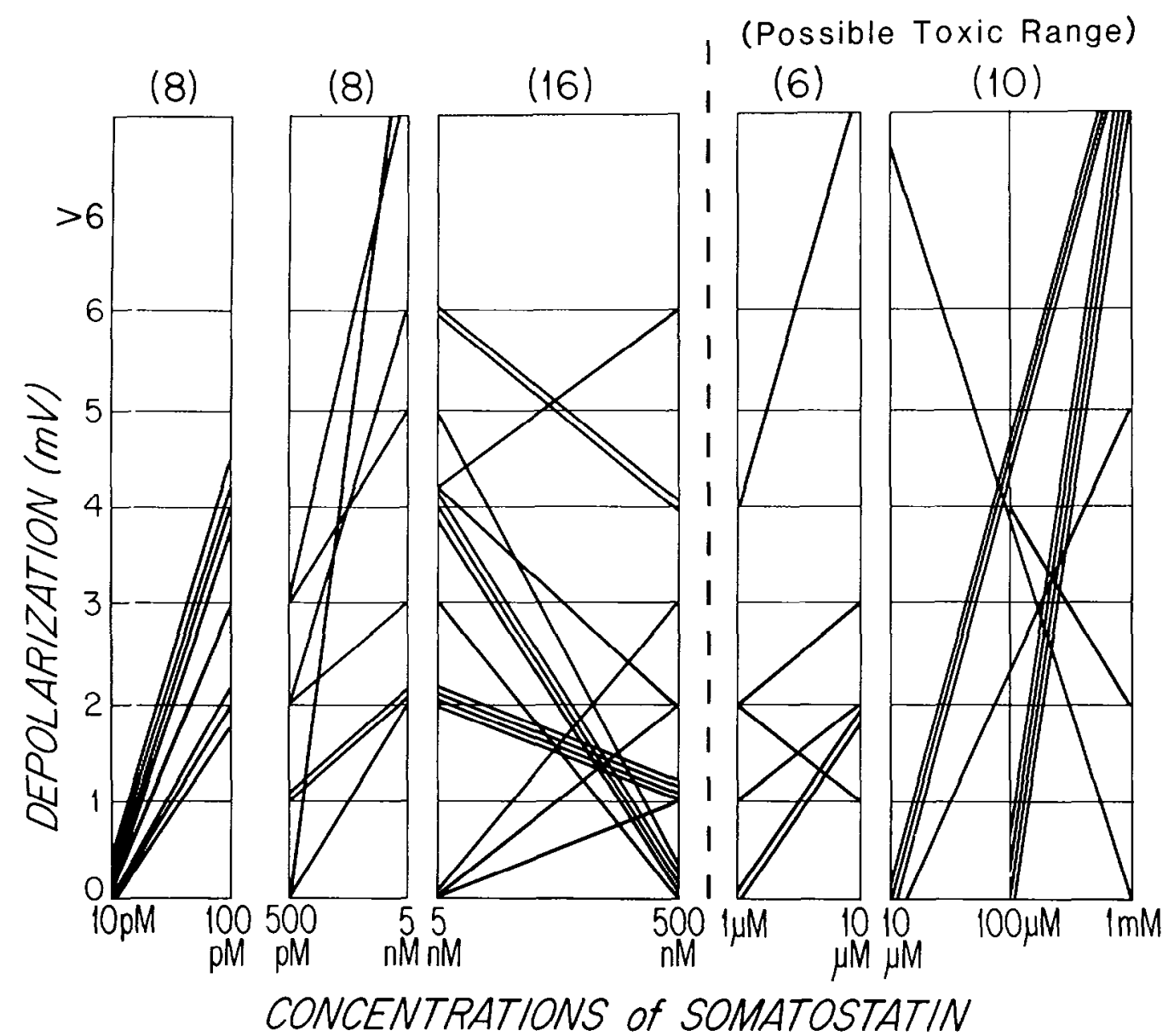

Figure 4. Dose pair comparison of the magnitude of depolarization evoked in the same neuron by application of two different concentrations of somatostatin. Each slanted line represents data from the intracellular recording from an individual neuron. There was a consistent increase in the magnitude of the depolarization response as the applied concentration was increased from 10 to $100 \mathrm{pM}$ and from $500 \mathrm{pM}$ to $5 \mathrm{nM}$, but as the concentration was increased from 5 to $500 \mathrm{nM}$ a smaller response was seen with the application of the higher concentration in 12 of 16 neurons. As concentrations were increased to greater than $1 \mu \mathrm{M}$, the higher concentrations were again associated with larger responses. The depolarizations seen in this third phase of the dose response relationship ( $1 \mu \mathrm{M}$ to $1 \mathrm{mM}$ ) often appeared qualitatively distinct from the depolarizations seen at lower concentrations and may represent toxic effects. Neurons were acceptable for inclusion if one of the two concentrations of somatostatin resulted in a depolarization of at least $1 \mathrm{mV}$. Concentrations are arranged on a repeated $l o g$ scale with spaces added to clarify the discontinuous nature of the data.

recordings and was seen to affect several different events. It follows that several different mechanisms of action may be involved. Nevertheless, the observation of inhibitory as well as excitatory effects is consistent with other published reports of both in vitro and in vivo studies and bears inclusion in the present report.

Dose-dependent excitation or inhibition. When the dosage range was extended to $10^{-3} \mathrm{M}$, inhibitory responses were seen in 15 of 159 recordings. In nine of these recordings, responses to a higher and a lower concentration were compared. Inhibition was seen with both of two different applied concentrations of somatostatin in only three of these recordings. However, in six of the nine recordings (three with effects on PSPs and three on APs) the inhibitory response was seen only with application of the higher of two somatostatin concentrations. In no case was an inhibitory response seen only with application of the lower of two concentrations.

In recordings in which at least one of two concentrations of somatostatin caused inhibition, four of nine recordings showed the lower concentration (5 to $100 \mathrm{nM}$ ) to cause excitation, whereas the higher concentration (50 nM to $10 \mu \mathrm{M})$ caused inhibition. This dose-dependent excitation or inhibition is illustrated by a recording in a neuron generating spontaneous APs (Fig. 10). A further phenomenon, illustrated by this recording, is that a neuron which initially responds to a relatively high concentration of somatostatin (500 nM in this case) with a decrease in activity or an inhibition can later, after considerable additional exposure to somatostatin, respond to that same concentration with an increase in activity or an excitation, similar to that seen earlier only with the lower concentration.

\section{Attempts to prevent desensitization by endogenous somatostatin}

One possible explanation for the relatively small depolarizations induced by somatostatin and for the fact that less than half of the neurons studied showed direct effects was that the neuronal responsivity was decreased by the relatively high concentrations of somatostatin in the culture growth medium, a consequence of somatosta- 


\section{LOW}
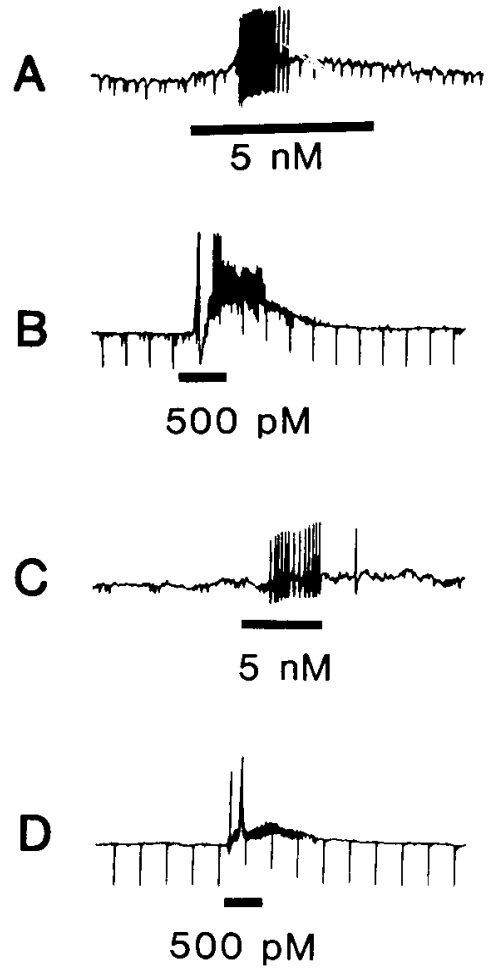

$\mathrm{HIGH}$
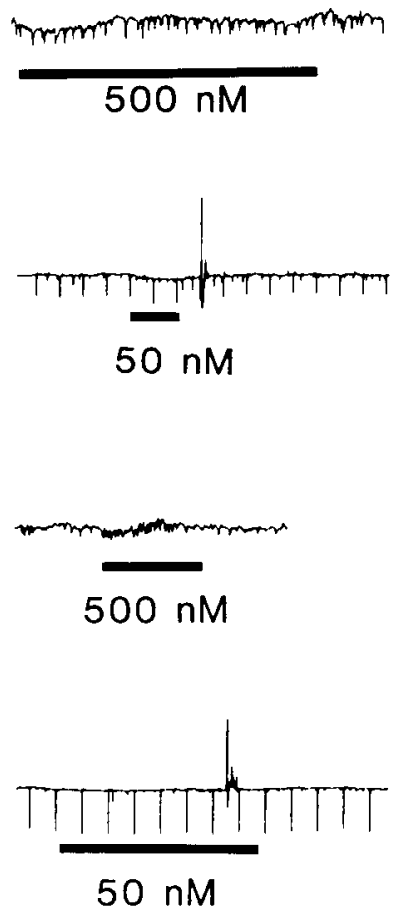

LOW
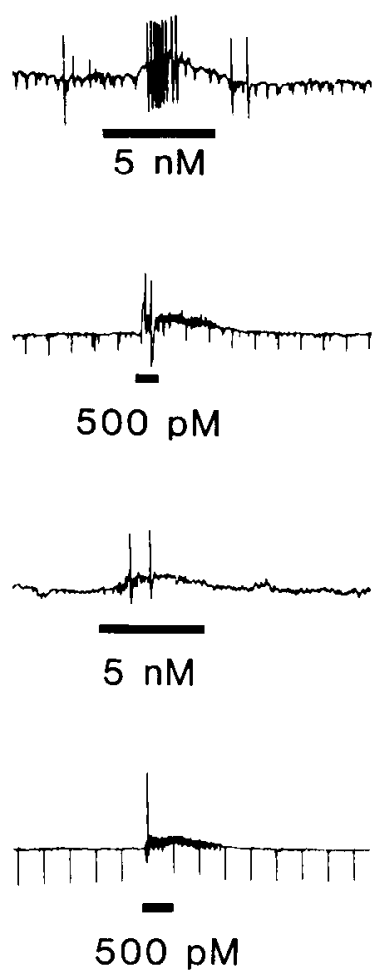

Figure 5. Larger responses of cortical neurons to the application of lower concentrations of somatostatin. A, Initial application of $5 \mathrm{nM}$ somatostatin to a neuron with spontaneous IPSPs causes significant depolarization and a burst of APs. Fifteen seconds later the application of $500 \mathrm{nM}$ causes only a small increase in IPSP frequency, but $10 \mathrm{sec}$ after this a repeat application of $5 \mathrm{~nm}$ results in a significant depolarization and burst of APs. $B$, Similar to $A$ except that there is no definite response to the higher concentration. $C$, Similar to $A$. $D$, The response to the $500 \mathrm{pm}$ somatostatin is a small burst of APs and a small depolarization associated with a run of EPSPs and a secondary decrease in membrane input resistance. Application of the higher concentration $(50 \mathrm{nM})$ is associated only with a delayed AP and small burst of EPSPs, whereas reapplication of 500 pM results in a response much more like the initial application and is significantly larger than that elicited by the intervening larger concentration. (In $B$ and $D$, voltage responses to hyperpolarizing pulses of current reflect a decreased $R_{\text {in }}$ with PSP response, an effect not seen with somatostatin in the absence of other factors to account for this change.) Values for $V_{m}:-50,-52,-68$, and $-60 \mathrm{mV}$, respectively.

tin production and release by the neurons in culture (Delfs et al., 1980). In the present study, the concentration of somatostatin in the culture growth medium ranged between 55 and 114 pM (Delfs et al, 1980), the latter concentration being higher than the usual threshold values for a neuronal response. Two techniques were used to decrease these levels in an attempt to avoid a possible chronic desensitization of neurons by the somatostatin released into the media: (1) TTX was used to inhibit release from the neurons, and (2) the cultures were maintained in the presence of high titers of antisomatostatin antibody in an effort to neutralize secreted somatostatin. All physiology studies were subsequently done in a phosphate-buffered physiological saline solution after removal of the culture growth medium.

In the first series of experiments, TTX was added to the usual culture medium at a final concentration of 3 $\times 10^{-7} \mathrm{M}$. Control cultures were maintained in the same medium without the TTX. At the end of $48 \mathrm{hr}$, the average concentration of somatostatin in the control medium was $73.9 \mathrm{pM}(n=12)$, whereas in the medium with TTX it was $5.8 \mathrm{pm}(n=6)$. However, despite this decreased exposure to somatostatin for $48 \mathrm{hr}$ prior to study, there was no change in the percentage of neurons showing either a depolarization or an increase in the frequency of PSPs, nor was there an alteration in the magnitude of these responses $(n=12)$.

In the second series of experiments, undiluted serum from a sheep immunized against cyclic somatostatin-14 (serum generously supplied by Seymour Reichlin) was substituted for the rat serum in the culture growth medium for $48 \mathrm{hr}$ prior to study $(n=13)$. Nonimmune sheep 
serum was used in the medium for control cultures $(n=$ 27 ). There was no change in the responsiveness of neurons in those cultures maintained in the anti-somatostatin antibody for $48 \mathrm{hr}$ prior to study. These two experiments suggest that chronic desensitization is not a major cause of unresponsiveness to somatostatin in the cultured cortical neurons.

\section{Discussion}

This study demonstrates that somatostatin is a predominantly excitatory agent when applied to rat cortical neurons in cell culture. Approximately one-third (34\%) of the tested neurons exhibited small depolarizations, often associated with an increase in membrane excitability, in response to the application of somatostatin. A larger fraction of the neurons (58\%) exhibited an increase in incoming spontaneous synaptic activity. These two categories are not mutually exclusive, and a single neuron could both undergo an apparently direct depolarization and also receive an increased frequency of incoming PSPs. Other effects of somatostatin included decreases in IPSP amplitudes and occasional inhibitory responses. We also describe several unusual aspects of the responses of cortical neurons to somatostatin. These include tachyphylaxis, an inverted U-shaped dose response curve for the depolarizing response, and a concentration-dependent excitation or inhibition.

Depolarization and excitation. Our findings that somatostatin was predominantly an excitatory agent are consistent with several investigations in situ as well as in vitro. Ioffe et al. (1978) found an increased firing rate in $58 \%$ of neurons in rabbit sensorimotor cortex in response to iontophoretic application of somatostatin, and Olpe et al. (1980) found an increase in firing rate in approximately $60 \%$ of rat cortical neurons. In frog spinal cord, Nicoll (1978) described an enhancement of the polysynaptic excitation of motor neurons generated by dorsal root stimulation, and Padjen (1977) reported an increase in size of ventral root potentials obtained by dorsal root stimulation. Using intracellular recording techniques, Macdonald and Nowak (1981) and Dodd and Kelly (1978) have reported that somatostatin depolarizes and excites
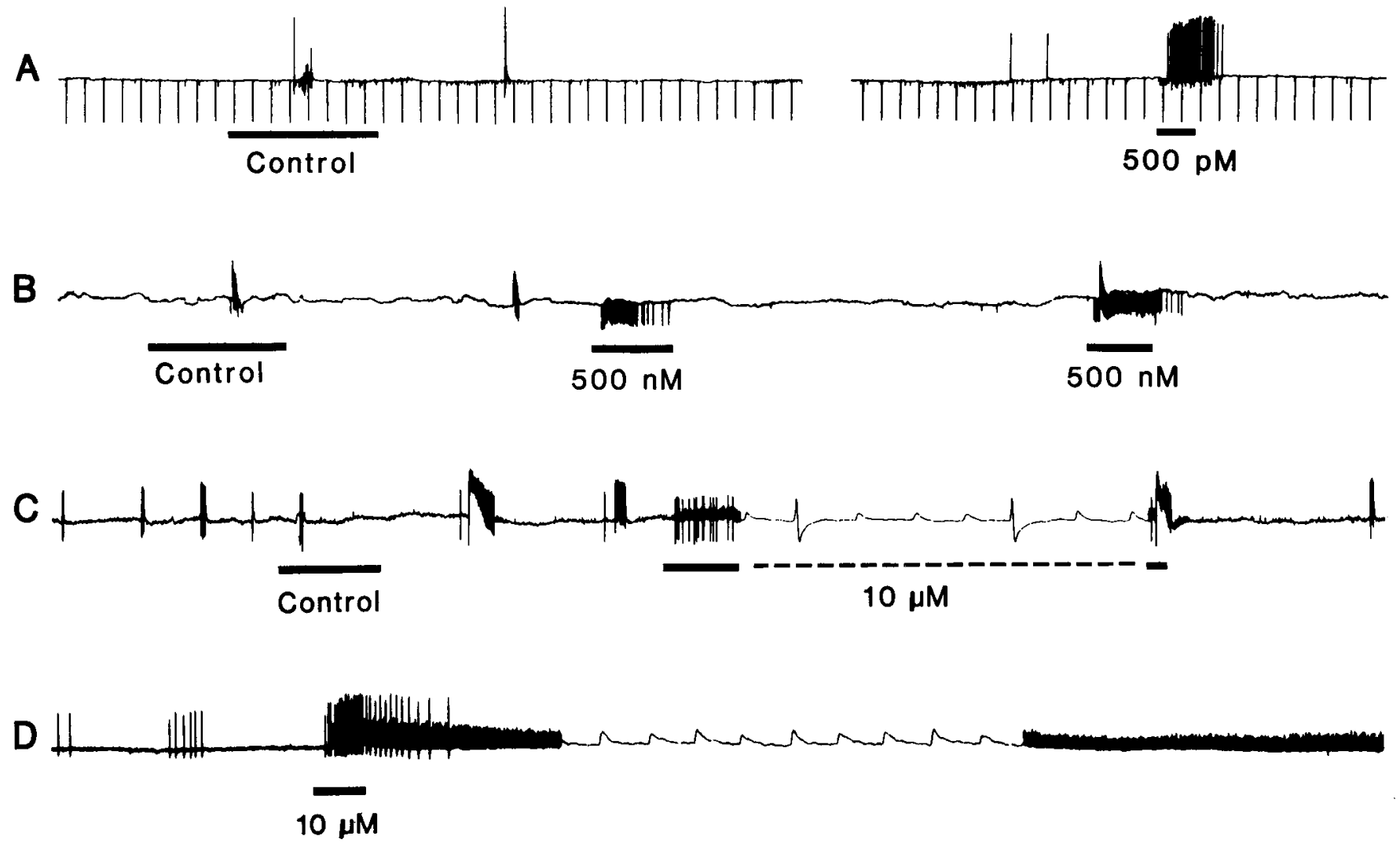

\section{$10 \mathrm{sec} 20 \mathrm{mV}$}

Figure 6. Somatostatin application resulting in increased incoming spontaneous synaptic activity in cortical neurons in the absence of any obvious direct membrane response. $A$, Neuron with a base line activity of intermittent small IPSPs, occasional EPSPs, and occasional APs. Somatostatin application results in a volley of incoming synaptic activity made up of first an increase in the frequency of the IPSPs and then the addition of high frequency EPSPs and associated APs. The larger downward deflections in this tracing are due to the regular injection of hyperpolarizing current (current trace not shown). $B$, In a neuron with only rare bursts of EPSPs and IPSPs, somatostatin applications result in brief periods of increased-frequency IPSPs. $C$, In another neuron the response consists of an increase in frequency of EPSPs, some of which evoke intermittent APs. Underlying EPSPs are more obvious with increased chart speed. $D$, The PSP response is initially obscured by a secondary increase in APs, but the basis of the response is clearly seen to be EPSPs when chart speed is increased. Resting $V_{m}:-56,-70$, and $-72 \mathrm{mV}$, respectively. AP heights are significantly attenuated by the penwriter. 
mouse spinal cord neurons and hippocampal pyramidal neurons, respectively.

The mechanism by which somatostatin produces depolarization is not yet known. It seems to be a direct membrane effect because it is not prevented by TTX. However, the possibility that somatostatin is causing the release of endogenous excitatory neurotransmitter at nerve terminals cannot be excluded. The decrease in membrane conductance and in the afterhyperpolarization seen in some recordings suggests that somatostatin may be decreasing a potassium conductance. Such an effect has been suggested in mouse spinal cord neurons in culture (Macdonald and Nowak, 1981).

Increased synaptic activity. There are several possible explanations for the increase in spontaneous PSPs. Somatostatin may either directly excite responsive neurons presynaptic to the recorded neuron or may act at presynaptic terminals to increase transmitter release. It also might act by decreasing inhibitory input to both excitatory and inhibitory neurons, a possibility that is consistent with the observed decrease in the amplitude of IPSPs. Alternatively, somatostatin might affect the postsynaptic membrane to augment PSPs and thus allow detection of PSPs too small to be distinguished from the base line before somatostatin application. However, an increase in the amplitude of IPSPs was never observed, and, although an increase in the amplitude of EPSPs was occasionally seen, this was an unusual effect of somatostatin application. Thus, it seems unlikely that somatostatin was simply increasing the size of PSPs.

Decreased amplitude of IPSPs. The simultaneous decrease in IPSP amplitude and increase in $R_{\text {in }}$ makes it unlikely that this effect on IPSPs is due to membrane shunting. It also raises the possibility that somatostatin causes a decrease in both a passive and a GABA-activated membrane conductance. However, whether this effect on IPSPs is due to a presynaptic effect on axon terminals or to an action of somatostatin on the postsynaptic receptor complex cannot be determined from the present data.

Heterogeneity of response. Our results indicate a heterogeneity of response to somatostatin. Only approximately $40 \%$ of the neurons demonstrated direct membrane effects (depolarization or increased excitability), whereas almost $60 \%$ of the recordings showed an increase in PSP frequency. In $10 \%$ of the recordings the response to somatostatin was entirely inhibitory, and in many other recordings the response was complex in that it was qualitatively different with different concentrations of somatostatin or with repeated exposure. This variability may be related to the heterogeneity of cell types and the complexity of local circuits within the cortical cultures. However, the type or magnitude of the response of an individual neuron was not predictable on a morphological

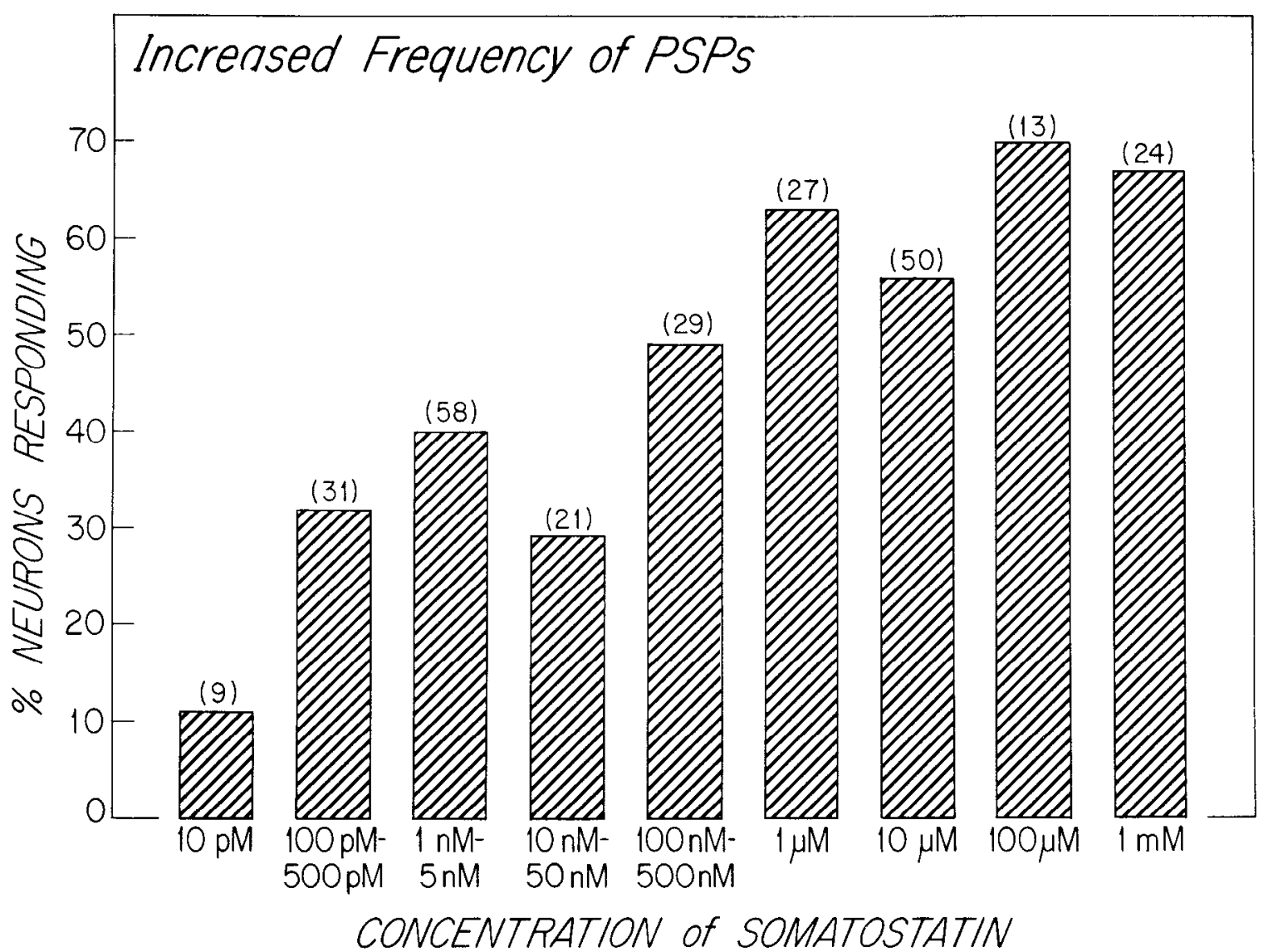

Figure 7. Percentage of tested neurons in which an increase in the frequency of PSPs was observed in response to the application of somatostatin in concentration groupings from $10 \mathrm{pM}$ to $1 \mathrm{mM}$. The number of neurons tested in each concentration range is noted in parentheses. The data suggest a monophasic dose response relationship with concentration changes over nine orders of magnitude. 

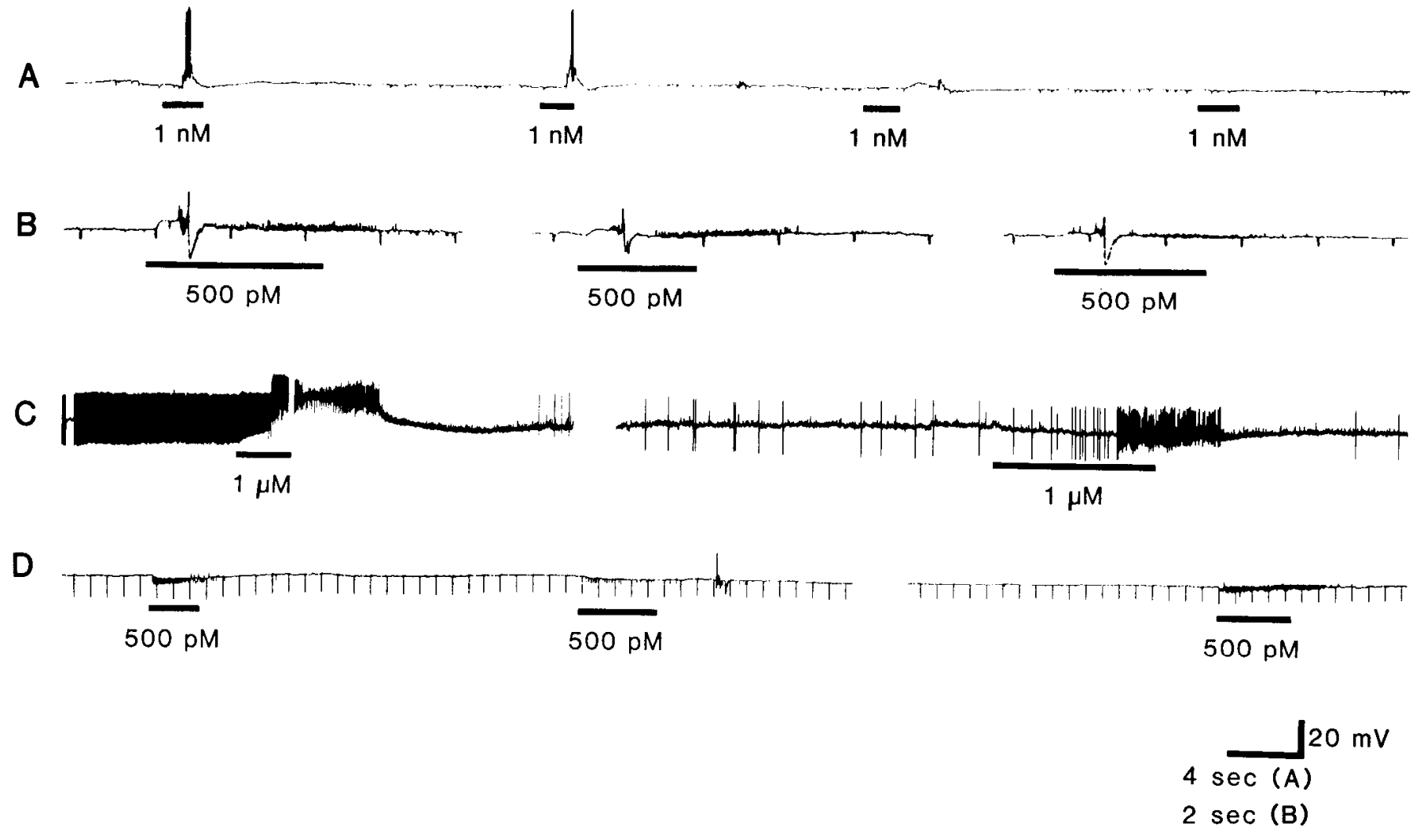

$10 \sec (C, D)$

Figure 8. Tachyphylaxis, or progressive diminution of the neuronal response to repeated applications of somatostatin. All traces are penwriter records of in tracellular recordings. The occurrence of APs and PSPs is easily monitored, although AP height is attenuated by the penwriter. $A$, Somatostatin application initially results in an $8-\mathrm{mV}$ depolarization and burst of APs, whereas successive applications are associated with a progressively diminished response until with the fourth application there is no detectable response at all. $R$, Two discernible responses to somatostatin in this recording are a rapid membrane depolarization and a later increase in frequency of incoming EPSPs. (During the PSP response there is a brief burst of APs with a hyperpolarizing afterpotential.) With repeated application there is a progressive diminution in both the direct membrane effect and the indirect increase in synaptic activity. $C$, In this neuron with initially rapidly firing APs, the response to the first application of somatostatin is a marked membrane depolarization, but the depolarizing response is completely absent with the second application when only a long latency increase in APs and underlying EPSPs is seen. $D$, Somatostatin application results in a burst of IPSPs and a secondary slight hyperpolarization of the membrane. This response is markedly diminished with a second application, but after a 4-min rest period there is a restoration of the original response. Regularly spaced hyperpolarizing potentials are due to injections of hyperpolarizing current. (Current trace not shown.) Values for $V_{m}:-65,-48,-56$, and $-50 \mathrm{mV}$, respectively.

basis, nor did it appear to be directly related to other parameters such as the $V_{\mathrm{m}}$, the $R_{\mathrm{in}}$, or the amount or type of ongoing spontaneous activity. This response variability also seemed not to reflect impurities in different batches of somatostatin, nor was it obviously related to methodological problems.

Published reports of the neuronal effects of somatostatin have been marked by variability and conflicting findings. Although most investigators have observed excitatory effects as noted above, a number of other workers have reported that somatostatin decreased neuronal firing. These reports of inhibitory effects have included studies in cerebral cortex, cerebellum, and hypothalamus of rats (Renaud et al., 1975) and in the dorsal horn of spinal cord in cats (Miletic et al., 1977). In another instance, two different groups, both doing intracellular studies in rat hippocampal slice preparations but using different methods of application of somatostatin, reported essentially opposite findings. One group applied somatostatin by iontophoresis, direct pressure injection, or local application of small droplets and reported neuronal depolarization and excitation (Dodd and Kelly, 1978). The other group applied somatostatin by bath perfusion and noted hyperpolarization and inhibition (Pittman and Siggins, 1981). Katayama and North (1980) recorded in the peripheral nervous system from myenteric plexus neurons in guinea pig and noted that somatostatin could cause either excitatory or inhibitory responses in a single cell, depending on the method of application. Our findings may provide a framework with which to begin to analyze these various results.

Tachyphylaxis. The phenomenon of a progressive diminution of responses to repeated applications of the same concentration of somatostatin seems to be a major influence on the magnitude of the response of the neurons in this sytem. It can occur with very brief exposures to low concentrations of this peptide and can readily obscure the neuronal effects of somatostatin altogether, especially the membrane depolarization and excitation. The mechanism by which tachyphylaxis occurs is unknown. 
Inverted $U$-shaped dose response curve. Our data also demonstrate that in individual neurons somatostatin causes a greater effect at a lower concentration than at a higher concentration over a specific range. The amplitude of the reversible depolarization seen with application of somatostatin in the picomolar to nanomolar range, as well as its likelihood of occurrence, peaks with the high picomolar to low nanomolar concentrations, whereas concentrations in the high nanomolar to low micromolar yield fewer and smaller depolarizing responses.

Several possibilities exist as mechanisms for this inverted U-shaped somatostatin dose response curve. One possibility is that a higher concentration of ligand may cause the receptor to be fixed in a noneffector confor-

A
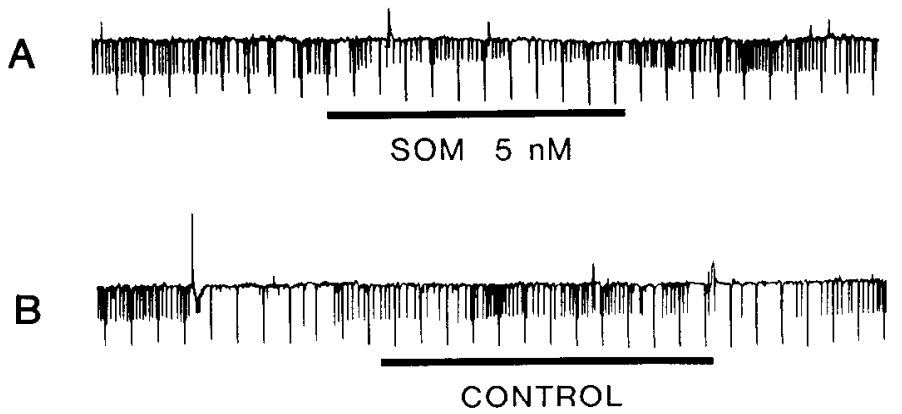

$\frac{}{5 \mathrm{sec}} 20 \mathrm{mV}$

Figure 9. Somatostatin ducreases the amplitude of IPSPs concomitant with an increase in the $R_{\text {in. }} A$, Amplitude of the IPSPs is decreased from an average of $12 \mathrm{mV}$ to an average of $7 \mathrm{mV}$ by the application of somatostatin, whereas the $R_{\text {in }}$ changes from 89 megohms to 96 megohms. These alterations are reversible with termination of the application. $B$, Control perfusion results in no significant change of either IPSP amplitude or of $R_{\text {in }}$. Resting $V_{m}$ is $-64 \mathrm{mV}$. mation. More sophisticated receptor binding studies may shed light on this possibility, but such a phenomenon might only be demonstrable under dynamic conditions where physiological responses are measurable. Another possibility is that higher concentrations of somatostatin could alter the cellular metabolism in a way that blocks the effect of the activated somatostatin receptor.

Toxic effects at higher doses. In the analysis of the dose response characteristics of somatostatin on cortical neurons we have chosen to consider the depolarizations seen with the application of high micromolar concentrations as nonphysiological responses. The large, long lasting depolarizations and protracted decreases in $R_{\text {in }}$ suggest that micromolar concentrations may cause effects by damaging the neuronal membrane. Nemeth and Cooper (1979) have reported that somatostatin applied to rat hippocampal synaptosomes at micromolar concentrations causes an efflux of the cytoplasmic enzyme lactate dehydrogenase ( $\mathrm{LDH})$, consistent with nonspecific membrane toxicity at these higher concentrations. It is possible that these toxic effects are preventing the physiological response to somatostatin and that this is responsible for the apparent decrease in response at higher concentration ranges.

Dose-dependent excitation or inhibition. Another possibility for the decreased responsiveness in the high nanomolar concentration range is a superimposed inhibitory action that is opposing the excitatory action. Our data suggest that lower concentrations of somatostatin produce only excitatory responses (often with membrane depolarization), whereas higher concentrations applied to the same neurons are more likely to have no effect or to produce inhibitory responses (with no change in membrane potential). These observations suggest the possibility that somatostatin is interacting with two different populations of receptors. At low concentrations, a predominant interaction with high affinity receptors could
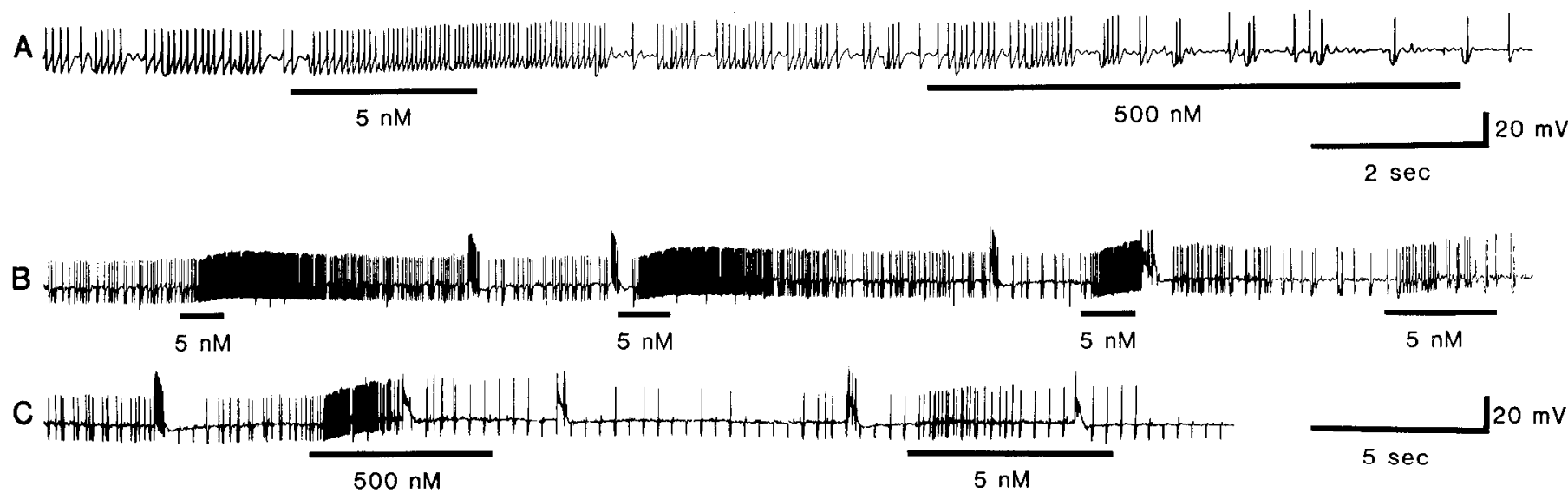

Figure 10. Qualitative alteration of the dose response relationship in a single cortical neuron with serial exposures to somatostatin. $A$, Neuron with ongoing regular firing of spontaneous APs. Application of 5 nM somatostatin results in an increased firing rate. The application of $500 \mathrm{~nm}$ somatostatin results in an inhibition of spontaneous firing. The APs remaining are those evoked as anode break spikes at the termination of each pulse of hyperpolarizing current. $B$, Same neuron 5 min after recording shown in $A$, so that spontaneous activity has now returned. Note slower chart speed. Somatostatin at 5 nm again results in a marked increase in firing rate, but repeated applications of this concentration are associated with a progressive diminution in response, with a gradually decreasing rate of spontaneous firing. $C$, After $3 \mathrm{~min}, 500 \mathrm{~nm}$ somatostatin is again applicd, but unlike the previous inhibitory response shown in $A$, the response is now a marked increase in the firing rate. The response to the 5 nm continues to be diminished compared to the responses to earlier applications. Resting $V_{m}$ is $-54 \mathrm{mV}$. Regularly spaced resistance monitoring pulses continue throughout the recording (current trace not shown). 
result in depolarization and excitation; whereas, at higher concentrations, an additional interaction with lower affinity receptors mediating inhibition could oppose the depolarizing-excitatory effects and prevent an observable membrane response. Inhibitory effects may occasionally be observed when the concentration is sufficiently high to bring the balance in favor of the inhibitory effect or when the excitatory component has decreased due to tachyphylaxis. Such a double action has been described for the cholinergic synapse in Aplysia (Wachtel and Kandel, 1971).

Among the major issues remaining unanswered is whether the physiologically relevant effect of somatostatin is the acute change in membrane excitability (with a concomitant increase in local synaptic activity), such as reported in this paper, or rather some more chronic effect, be it metabolic or electrophysiological, that might remain relatively unnoticed under the paradigm of short term monitoring with intracellular recordings. Nevertheless, it is of possible relevance to ask how our findings of prominent excitatory effects of somatostatin may relate to its actions within the endocrine and gastrointestinal systems where it is generally regarded as a completely inhibitory compound. For instance, the local activity of a low concentration of somatostatin might under some conditions be to function as a releasing factor for substances whose release it is now believed to solely inhibit. One report has noted that small doses of intraventricular somatostatin increase growth hormone release in rats, and indeed the smaller dose was noted to cause a larger increase in growth hormone (Lumpkin et al., 1981). Our findings raise the possibility that somatostatin might also function in these other systems as an excitatory influence.

It seems likely that the dose response characteristics described for the effects of somatostatin on cortical neurons in culture will also be found in other neuronal systems. Therefore, an awareness of these phenomena will be important in the design of further studies with somatostatin. Tachyphylaxis alone is a factor which could easily obscure the results of experimental investigations and biphasic dose response curves can add considerable complexity to data interpretation. Of at least equal importance, these phenomena may well be operant in the regulation of neuronal responses in intact organisms. An understanding of them and the underlying processes they represent will be important in an understanding of the neuronal effects of somatostatin.

\section{References}

Berelowitz, M., J. Matthews, B. L. Pimstone, S. Kronheim, and H. Sacks (1978) Immunoreactive somatostatin in rat cerebral cortical and hypothalamic synaptosomes. Metabolism 27(Suppl. 1): 1171-1173.

Brazeau, P, W. Vale, R. Burgus, N. Ling, M. Butcher, J. Rivier, and R. Guillemin (1973) Hypothalamic polypeptide that inhibits the secretion of immunoreactive pituitary growth hormone. Science 179: 77-79.

Davies, P., R. Katzman, and R. D. Terry (1980) Reduced somatostatin-like immunoreactivity in cerebral cortex from cases of Alzheimer disease and Alzheimer senile dementia. Nature 288: 279-280.

Delfs, J. R., and M. A. Dichter (1981) Effects of somatostatin on cortical neurons in culture: Membrane effects with unu- sual dose-response characteristics. Soc. Neurosci. Abstr. 7: 429.

Delfs, J. R., M. A. Dichter, R. Robbins, J. Connolly, and S. Reichlin (1979) Somatostatin in rat cortical neurons in cell culture: Synthesis and physiologic effects. Soc. Neurosci. Abstr. 5: 526.

Delfs, J., R. Robbins, J. Connolly, M. Dichter, and S. Reichlin (1980) Somatostatin production by rat cerebral neurones in dissociated cell culture. Nature 283: 676-677.

Dichter, M. A. (1978) Rat cortical neurons in cell culture: Culture methods, cell morphology, electrophysiology, and synapse formation. Brain Res. 149: 279-293.

Dodd, J., and J. S. Kelly (1978) Is somatostatin an excitatory transmitter in the hippocampus? Nature 273: 74-675.

Havlicek, V., M. Rezek, and H. Friesen (1975) Somatostatin and thyrotropin releasing hormone: Central effect on sleep and motor system. Pharmacol. Biochem. Behav. 4: 455-459.

Ioffe, S., V. Havlicek, H. Friesen, and V. Chernick (1978) Effect of somatostatin (SRIF) and L-glutamate on neurons of the sensorimotor cortex in awake habituated rabbits. Brain Res. 153: 414-418.

Iversen, L. L., S. D. Iversen, F. Bloom, C. Douglas, M. Brown, and W. Vale (1978) Calcium-dependent release of somatostatin and neurotensin from rat brain in vitro. Nature 273: 161163.

Katayama, Y., and R. A. North (1980) The action of somatostatin on neurones of the myenteric plexus of the guinea-pig ileum. J. Physiol. (Lond.) 303: 315-323.

Krulich, L., A. P. S. Dhariwal, and S. M. McCann (1968) Stimulatory and inhibitory effects of purified hypothalamic extracts on growth hormone release from rat pituitary in vitro. Endocrinology 83: 783-790.

Lee, S. L., V. Havlicek, A. E. Panerai, and H. G. Friesen (1979) High $\mathrm{K}^{+}$-induced release of somatostatin from the cortical preparation of rat brain. Experientia 35: 351-352.

Lumpkin, M. D., A. Negro-Vilar, and S. M. McCann (1981) Paradoxical elevation of growth hormone by intraventricular somatostatin: Possible ultrashort-loop feedback. Science 211: 1072-1074.

Macdonald, R., and L. Nowak (1981) Somatostatin has excitatory actions on murine spinal cord neurons in primary dissociated cell culture. Soc. Neurosci. Abstr. 7: 429.

Martin, J. B., P. Brazeau, G. S. Tannenbaum, J. O. Willoughby, J. Epelbaum, L. C. Terry, and D. Durand (1978) Neuroendocrine organization of growth hormone regulation. In The Hypothalamus, S. Reichlin, R. J. Baldessarini, and J. B. Martin, eds., pp. 329-355, Raven Press, New York.

Miletic, V., M. S. Kovacs, and M. Randic (1977) Actions of somatostatin and methionine-enkephalin on cat dorsal horn neurons activated by noxious stimuli. Soc. Neurosci. Abstr. 3: 488 .

Nemeth, E. F., and J. R. Cooper (1979) Effect of somatostatin on acetylcholine release from rat hippocampal synaptosomes. Brain Res. 165: 166-170.

Nicoll, R. A. (1978) Peptide receptors in the CNS: Neurophysiologic studies in neurobiology of peptides. Neurosci. Res. Program Bull. 16: 272-285.

Olpe, H. -R., V. J. Balcar, H. Bittiger, H. Rink, and P. Sieber (1980) Central actions of somatostatin. Eur. J. Pharmacol. 63: 127-133.

Padjen, A. L. (1977) Effects of somatostatin on frog spinal cord. Soc. Neurosci. Abstr. 3: 411.

Patel, Y. C., and S. Reichlin (1978) Somatostatin in hypothalamus, extrahypothalamic brain, and peripheral tissues of the rat. Endocrinology 102: 523-530.

Pittman, Q. J., and G. R. Siggins (1981) Somatostatin hyperpolarizes hippocampal pyramidal neurons in vitro. Brain Res. 121: 402-408. 
Plotnikoff, N. P., A. J. Kastin, and A. V. Schally (1974) Growth hormone release inhibiting hormone: Neuropharmacologic studies. Pharmacol. Biochem. Behav. 2: 693-696.

Renaud, L. P., J. B. Martin, and P. Brazeau (1975) Depressant action of TRH, LH-RH and somatostatin on activity of central neurones. Nature 255: 233-235.

Rezek, M., V. Havlicek, K. R. Hughes, and H. Friesen (1977) Behavioral and motor excitation and inhibition induced by the administration of small and large doses of somatostatin into the amygdala. Neuropharmacology 16: 157-162.

Snodgrass, S. R., W. F. White, B. Biales, and M. Dichter (1980)
Biochemical correlates of GABA function in rat cortical neurons in culture. Brain Res. 190: 123-138.

Srikant, C. B., and Y. C. Patel (1981a) Somatostatin receptors: Identification and characterization in rat brain membranes. Proc. Natl. Acad. Sci. U. S. A. 78: 3930-3934.

Srikant, C. B., and Y. C. Patel (1981b) Somatostatin analogs. Dissociation of brain receptor binding affinities and pituitary actions in the rat. Endocrinology 108: 341-343.

Wachtel, H., and E. R. Kandel (1971) Conversion of synaptic excitation to inhibition at a dual chemical synapse. J. Neurophysiol. 34: 56-68. 